\title{
Meter Reading Scheme Based on Automatic Switching between Two Local Communication Channels
}

\author{
Guiping Deng ${ }^{1, a}$, Tiejie Wang ${ }^{2, b}$ and Miao Zhou ${ }^{3, c}$ \\ ${ }^{1}$ State Grid Hubei Electric Power Research Institute, Wuhan, 430077, China \\ ${ }^{2}$ Wuhan Electric Power Technical College, Wuhan, 430079, China \\ ${ }^{3}$ State Grid Hubei Electric Power Research Institute, Wuhan, 430077, China \\ aemail: smile1248@sohu.com, ${ }^{b}$ email:wangtiejie@163.com, ${ }^{c}$ email:34049812@qq.com
}

\begin{abstract}
Keywords: power line carrier, micro-power wireless, two local communication channels, automatic switching, electric energy data acquire system.

Abstract. This paper presented the meter reading scheme based on automatic switching between two local communication channels of power line carrier and micro-power wireless. Firstly, the structure of the meter reading system based on automatic switching between two local channels of power line carrier and micro-power wireless was introduced. Then two key technologies to realize this scheme which are two local channels automatic switching technology and Ad Hoc network technology are presented. The high communication success rate and accuracy of this meter reading scheme provide effective technical support for the construction of efficient, stable and practical electric energy data acquire system.
\end{abstract}

\section{Introduction}

The electric energy data acquire system is an important component of realizing the information interaction between the power supply company and electric power users. Intact local communication channel plays a key role in the reliable operation of the electric energy data acquire system[1,2]. Currently, the electric energy data acquire system in China mainly adopts low voltage power line carrier or micro-power wireless communication mode[3,4]. With the large-scale construction and application of the electric energy data acquire system, the single channel local communication mode has met some insurmountable technical problems from the system operation[5]. The diversity of the system applications has also put forward higher requirements to the local communication, while these higher requirements are difficult to be satisfied by the single local channel communication mode.

This paper presented the meter reading scheme based on automatic switching between two local communication channels of power line carrier and micro-power wireless. The two channels of power line carrier and micro-power wireless coordinate and supply each other, and each of them plays the advantages of itself, which effectively guarantees the local communication stability of the electric energy data acquire system and realizes the higher meter reading success rate and accuracy.

\section{Framework of Meter Reading Scheme with Two local Communication Channels}

As shown in Fig.1, the system includes: system master station, the remote GPRS or CDMA communication channels, concentrator, the two local communication channels of power line carrier and micro-power wireless and smart meters. The data transmission between the master station and the concentrator is based on the remote communication channel, while the data transmission between the concentrator and smart meters is through the two local communication channels.

The key to realize the two local channels meter reading is the two local channels communication technology. The key two aspects of technical difficulties are how the two local channels to switch during communication and how to route and organize network in this two local channels meter reading system. In the following, this paper will focus on the automatic switching design and Ad Hoc network design of the two local channels meter reading scheme. 


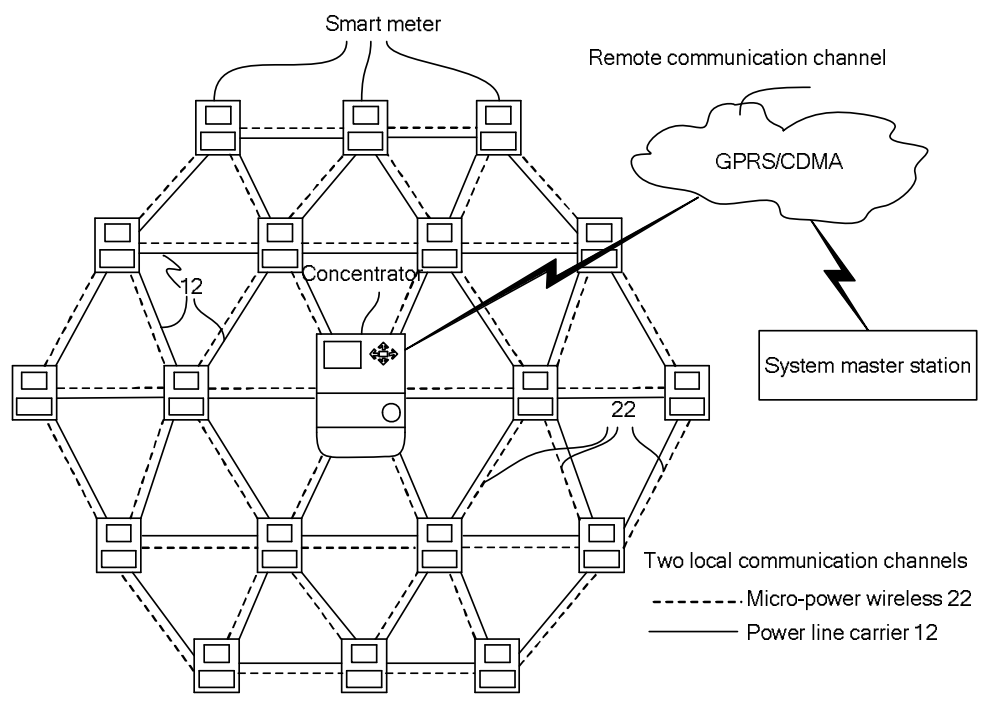

Fig.1. Framework diagram of the electric energy data acquire system with two local communication channels of power line carrier and micro-power wireless

\section{Automatic Switching Design between Two Local Channels}

To realize automatic switching between two channels of the power line carrier and micro-power wireless, both the power line carrier channel received signal strength detection module and the micro-power wireless channel received signal strength detection module need to be designed. After both the hardware and software filtering separately for each channel received signal, each detection module calculated out respective average signal strength value for power line carrier channel or micro-power wireless channel. Then the communication quality of each channel can be judged by comparing the average signal strength value with the set threshold, which will determine the suitable one-in-two channel for current situation. Finally, the automatic switching between the two channels was realized.

The structure of power line carrier channel received signal strength detection module is given in Fig.2. The power line carrier source signal will be intervened by the outside noise when travelling through the carrier channel. A part of the noise can be filtered by the hardware filter, and a part of the noise can be filtered by the software filter. After the hardware and software filtering, the noise can be basically eliminated. Then the received signal strength average value for power line carrier channel is measured by the detector. After travelling through the power line carrier channel, the measured received signal strength average value for power line carrier channel is between $-90 \mathrm{dbm}$ and $-10 \mathrm{dbm}$. If this average value is less than $-55 \mathrm{dbm}$, it shows that the communication quality of the current power line carrier channel is very poor and cannot communicate normally. If this average value is between $-55 \mathrm{dbm}$ and $-50 \mathrm{dbm}$, it means that the communication quality of the current power line carrier channel is of average and can be used as an alternative channel. If this average value is more than -50dbm, it means that the communication quality of the current power line carrier channel is very good. The value of $-50 \mathrm{dbm}$ is generally treated as the critical point to judge the communication quality of the current carrier channel.

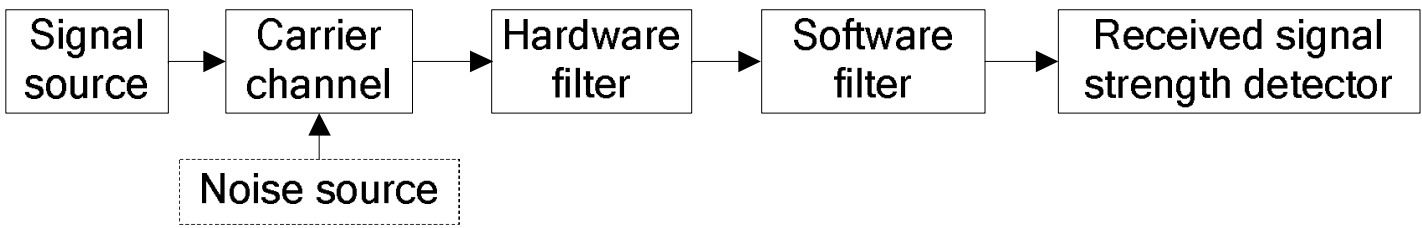

Fig.2. Structure of the received signal strength detection module for power line carrier channel

The structure of the received signal strength detection module for the micro-power wireless channel is shown in Fig.3. The micro-power wireless source signal will be intervened by the outside noise when 
travelling through the wireless channel. After the hardware and software filtering, those noise can be basically eliminated. Then the received signal strength average value for micro-power wireless channel is measured by the detector.

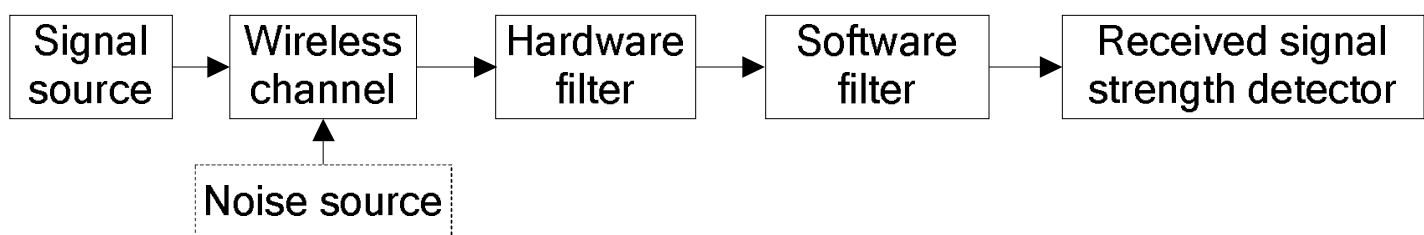

Fig.3. Structure of the received signal strength detection module for micro-power wireless channel

After travelling through the micro-power wireless channel, the measured received signal strength average value for micro-power wireless channel is between $-110 \mathrm{dbm}$ and $-30 \mathrm{dbm}$. If this average value is less than $-90 \mathrm{dbm}$, it shows that the communication quality of the current micro-power wireless channel is very poor and cannot communicate normally. If this average value is between $-90 \mathrm{dbm}$ and $-85 \mathrm{dbm}$, it means that the communication quality of the current micro-power wireless channel is of average and can be used as an alternative channel. If this average value is more than $-85 \mathrm{dbm}$, it means that the communication quality of the current micro-power wireless channel is very good. The value of $-85 \mathrm{dbm}$ is generally treated as the critical point to judge the communication quality of the current wireless channel.

The two detected received signal strength average values were compared with the preset threshold values by the channel selection module. Set the threshold W1 as $-85 \mathrm{dbm}$, W2 as $-90 \mathrm{dbm}, \mathrm{Z} 1$ as $-50 \mathrm{dbm}$ and $\mathrm{Z} 2$ as $-55 \mathrm{dbm}$.

(a) In the first case, if the received signal strength average value for micro-power wireless channel is greater than the threshold W1, the wireless channel will be chosen.

(b) The second case is under that the received signal strength average value for the wireless channel is between threshold W2 and threshold W1.

(b.1) When the received signal strength average value for the carrier channel is greater than the threshold Z1, the carrier channel will be chosen.

(b.2) When the received signal strength average value for the carrier channel is less than the threshold Z1, the wireless channel will be selected.

(c)The third case is under that the received signal strength average value for wireless channel is less than the threshold W2.

(c.1) When the received signal strength average value for the carrier channel is greater than the threshold Z2, the carrier channel will be chosen.

(c.2) When the received signal strength average value for the carrier channel is less than the threshold Z2, the two received signal strength average values respectively for carrier channel and wireless channel will be detected again after the delay.

The wireless channel is selected as the default preferred channel for its faster transmission speed compared with the carrier channel. According to the different cases mentioned above, if the current received signal strength of the two channels meets the case of (a) or (b.2), the wireless channel will be selected and there is no need to switch. If the current received signal strength of the two channels meets the case of (b.1) or (c.1), the carrier channel will be selected and the wireless channel will be switched to the carrier channel automatically at this time. And so on, the subsequent automatic channel switching will go on.

\section{Ad Hoc Network Design with Two Local Channels}

In the proposed two local channels meter reading scheme, there are four tasks in the network operation. The four tasks are network construction, network optimization, network repair and network reconstruction. All network nodes after site installation do not have network information at first. And the routing unit will start network construction task automatically. After the completion of the network construction, the entire routing information needs network optimization. This part mainly takes the 
advantages of the centralized network and does the optimization work in accordance with the previously established network structure. When congestion and local failure occur in the network, the adaptive listening part starts automatically working to repair the network. When the center node of the entire network moved or changed, it starts automatically to reconstruct the network.

In the single local channel meter reading scheme, when doing the network repair in the wireless or power line carrier Ad Hoc network technology, the network optimization and repair can only be completed through the master node, which leads to meter reading with low real time and accuracy. The two local channels meter reading scheme proposed in this paper adopts the Ad Hoc network technology, which doesn't need to go through the master node and automatically optimizes the previous communication link. When a slave node in the operating network fails to maintain communication because of blockage or partial failure, the adjacent nodes which share the same parent and child nodes with the disabled slave node will detect and listen to the failure news immediately. Since more than one adjacent node can listen to the news, so the comprehensive communication quality considering both upward and downward data transmission is used to select the optimal adjacent node. Because each node can detect the communication quality of own down link and record the relevant parameter values, the parent node of adjacent nodes can able to know the comprehensive communication quality considering both up and down link of all those adjacent nodes. According to the comprehensive communication quality, an optical up and down communication link and the adjacent node to realize this optical link are selected to respectively replace the failed communication link and node because of congestion or partial failure, which realizes the automatic network optimization for the previous network communication link.

\section{Conclusion}

The proposed meter reading scheme based on automatic switching between two local communication channels has been running on site for nearly six months. And the operation effect is very good. This scheme can complete the task of collecting frozen data in a very short period of time, and can achieve the $100 \%$ success rate of reading daily meter data. This proposed scheme takes the advantages of both the power line carrier meter reading system and the micro-power wireless meter reading system at the same time, which makes the system more reliable, accurate, real-time and efficient. These characteristics are of great significance for building the electric energy data acquire system for full coverage, full acquire and full bill control. According to application effect, the further improvement will be made for this proposed scheme to meet the needs of the electric energy data acquire system with increasing complexity and diversified applications.

\section{References}

[1] Ye Yan, Yi Qian and Sharif Hamid, "A Secure and Reliable In-network Collaborative Communication Scheme for Advanced Metering Infrastructure in Smart Grid," in Proceedings of 2011 IEEE Wireless Communications and Networking Conference (WCNC), USA, 2011.

[2] Baohui Lu and Yonghong Ma. Research on Communication System of Advanced Metering Infrastructure for Smart Grid and Its Data Security Measures[J]. Power System Technology, vol.37, No.8, pp.2244-2249, Aug.2013 (In Chinese).

[3] Jianhua Qian, Baichao Chen. An automatic meter reading system based on wireless communication[J]. Power System Technology, vol.28, No.5, pp.73-76, Mar.2004 (In Chinese).

[4] M. Y. Zhai. Transmission characteristics of low -voltage distribution networks in China under the smart grids environment[J]. IEEE Transactions on Power Delivery, 2011, 26(1): 173.

[5] Meng H, Guan Y L, Chen S. Modeling and analysis of noise effects on broadband power-line communications[J]. IEEE Transactions on Power Delivery, 2005, 20(2): 630-637. 